\title{
Effect of the Kapitza temperature jump on thermal processes in nanofluids
}

\author{
Sergey Novopashin, Marina Serebryakova ${ }^{a}$ and Gennadiy Sukhinin \\ Kutateladze Institute of Thermophysics, Lavrentyev Ave., 1, 630090 Novosibirsk, Russian Federation
}

\begin{abstract}
Two analytical solutions describing thermal processes in a nanofluid based on spherical nanoparticles taking into account the Kapitza temperature jump on a particle-fluid boundary were found. In the first solution the thermal conductivity of nanofluids was found with the help of Maxwell approach. The second solution describes stationary heat exchange between a spherical particle and fluid in two different conditions. A dimensionless criterion characterizing the effect of the Kapitza temperature jump on thermal processes in nanofluids has been obtained in both solutions.
\end{abstract}

\section{Introduction}

A temperature jump at an interface between two media was detected by Kapitza [1] in experiments on the thermal conductivity of superfluid helium. This phenomenon is often referred to as the Kapitza interfacial thermal resistance (KITR). Since the KITR is low, it is observed in systems having small dimensions or a high thermal conductivity. Until recently, it has been thought that the Kapitza temperature jump at room temperature can be disregarded [2]. However, the development of nanotechnologies initiated investigations of this phenomenon in a wide temperature range and for various pairs of solids [3,4]. The effect of the KITR on the thermal conductivity of solid composite materials was theoretically studied in [5]. The KITP at room temperature can vary in the range of $\mathrm{G}=$ $\left(2 \times 10^{-10}-2 \times 10^{-7}\right) \mathrm{m}^{2} \mathrm{~K} / \mathrm{W}$ [4]. The corresponding characteristic scale for copper (thermal conductivity is $\kappa \approx 400 \mathrm{~W} /(\mathrm{m} \mathrm{K}))$ is evaluated: $L=G \kappa \approx 10^{-7}-10^{-4} \mathrm{~m}$. The KITR at a fluid-solid interface was studied in [6]. The KITR can range from $3 \times 10^{-9}$ to $10^{-8} \mathrm{~m}^{2} \mathrm{~K} / \mathrm{W}$ when the surface of the solid is lyophilic or lyophobic to the given fluid. The thermal conductivity of fluids is much lower, e.g., the thermal conductivity of water is $\kappa \approx 0.6 \mathrm{~W} /(\mathrm{m} \mathrm{K})$. The characteristic scale at which the contribution of the KITR should be taken into account for, e.g., water is estimated as $L=G \kappa \approx(2 \times$ $\left.10^{-9}-6 \times 10^{-9}\right) \mathrm{m}$. The characteristic distance between particles in suspensions with nanoparticles (nanofluids [7]) with a characteristic volume content of about $1 \%$ is larger than the diameter of a particle by a factor of 3-5. Consequently, the Kapitza temperature jump can affect thermal processes in nanofluids. We indicate two thermal problems for which the presence of the Kapitza temperature jump can be important. The first problem is the effect of the KITR on the thermal conductivity of nanofluids. The second problem is heat transfer between a particle and a medium at the release of heat in nanoparticles, e.g., at hyperthermia with the heating of magnetic nanoparticles by an ac magnetic

$\overline{{ }^{e} \text {-mail: serebriakova.marina@gmail.com }}$

(C) The Authors, published by EDP Sciences. This is an open access article distributed under the terms of the Creative Commons Attribution License 4.0 (http://creativecommons.org/licenses/by/4.0/). 
field $[10,11]$ or at a phase transition in the material of a nanoparticle used for the intensification of heat transfer $[12,13]$.

\section{Thermal conductivity of nanofluids}

Let us consider a linear distribution of temperatures at infinity (constant gradient of temperature): $\vec{\nabla} T=\vec{A}=$ const $[14]$. Indices $\mathrm{p}$ and $\mathrm{f}$ will be used to label the values of a particle and fluids. Thus, the coefficients of thermal conductivity of these regions are $\kappa_{\mathrm{p}}$ and $\kappa_{\mathrm{f}}$, respectively. Let us assume that the radius of the particle is a. Applying standard boundary conditions, temperature equality and thermal flow continuity on contact surfaces, we find the distribution of temperatures using the following equations:

$$
T_{p}(\vec{r})=C_{p} \vec{A} \cdot \vec{r}, \quad(r<a) ; \quad T_{f}(\vec{r})=\left(1+C_{p} / r^{3}\right) \vec{A} \cdot \vec{r} \quad(r \geq a)
$$

Constants $\mathrm{C}_{\mathrm{p}}$ and $\mathrm{C}_{\mathrm{f}}$ are calculated with the help of conditions on the particle boundary:

$$
T_{p}(a)-T_{f}(a)=G q, \quad q=-\kappa_{p} \frac{\partial T_{p}}{\partial r}=-\kappa_{f} \frac{\partial T_{f}}{\partial r} \quad(r=a)
$$

Here $\mathrm{G}$ is the Kapitza Interfacial Thermal Resistance (KITP). Calculations give temperature distributions:

$$
T_{p}(\vec{r})=\frac{3 \kappa_{f}}{\kappa_{p}+2 \kappa_{f}\left(1+\kappa_{p} G / a\right)} \vec{A} \cdot \vec{r} ; \quad T_{f}(\vec{r})=\left(1+\frac{\kappa_{f}\left(1+\kappa_{p} G / a\right)-\kappa_{p}}{\kappa_{p}+2 \kappa_{f}\left(1+\kappa_{p} G / a\right)} \cdot \frac{a^{3}}{r^{3}}\right) \vec{A} \cdot \vec{r}
$$

Calculating heat conductivity of nanofluids we will use Maxwell approach [9]. Let nanofluid ocupy a spherical region with radius $R$ in the same base fluid. In this case temperature distribution in a base fluid in the accordance with (3) at $G=0$ will be as follows:

$$
T_{f}(\vec{r})=\left(1+\frac{\kappa_{f}-\kappa_{n p}}{\kappa_{n f}+2 \kappa_{f}} \cdot \frac{a^{3}}{r^{3}}\right) \vec{A} \cdot \vec{r},
$$

where $\kappa_{n f}$ is unknown heat conductivity. On the other hand, temperature distribution could be found as a sum of $\boldsymbol{n}$ spherical particles. Substituting $n a^{3}=\varphi R^{3}$, where $\varphi$ is volume fraction of nanoparticles, and introducing dimensionless paremeter $\gamma=G \kappa_{f} / a$, we get a final dependence:

$$
\frac{\kappa_{n f}}{\kappa_{f}}=\frac{1+2 \kappa_{f} / \kappa_{p}-2 \varphi\left(\kappa_{f} / \kappa_{p}-1\right)+2 \gamma(1-\varphi)}{1+2 \kappa_{f} / \kappa_{p}+\varphi\left(\kappa_{f} / \kappa_{p}-1\right)+\gamma(2+\varphi)}
$$




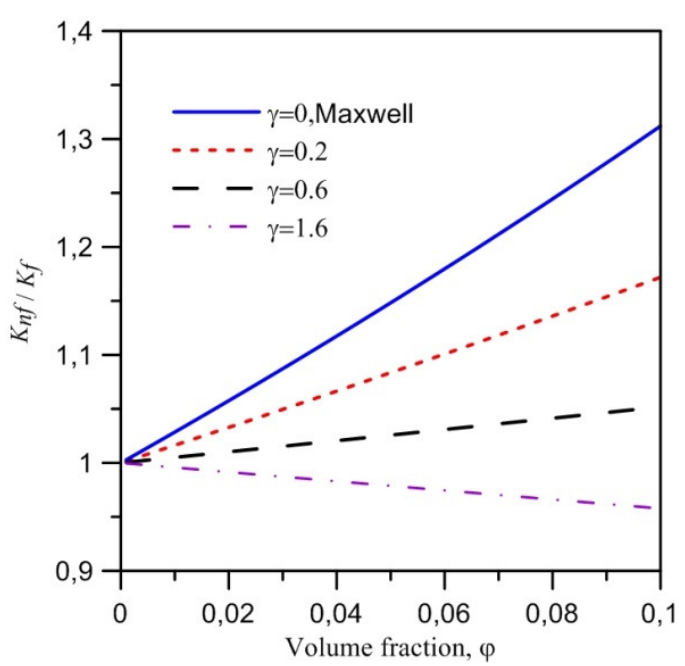

Figure1. The influence of KITP on thermal conductivity for a different value of parameter $\gamma\left(\kappa_{p}>>\kappa_{f}\right)$.

The analysis of the formulae (5) shows that for any value of $\gamma>0$ the KITP results in decreasing heat conductivity of nonofluids with respect to Maxwell's prediction. For practically important case $\kappa_{p}>>$ $\kappa_{f}$, one gets $\kappa_{n f}=\kappa_{f}(1+2 \varphi+2 \gamma(1-\varphi)) /(1-\varphi+\gamma(2+\varphi))$. Figure 1 shows the influence of KITP for different value of parameter $\gamma$. It is interesting to note that for the condition $\kappa_{p}>>\kappa_{f}$ and $\gamma=1$, $\kappa_{n f} \approx \kappa_{f}$.

\section{Heat exchange between a particle and a fluid}

We solve a steady-state inhomogeneous heat conduction equation taking into account the Kapitza temperature jump at the boundary of a spherical particle of radius $\boldsymbol{a}$ under two different conditions. First, a heat source $Q=$ const inside the spherical particle and temperature at infinity $\boldsymbol{T}_{\infty}$ is given. Second, the temperature of the particle $\boldsymbol{T}_{\boldsymbol{p}}$ and the temperature at infinity $\boldsymbol{T}_{\infty}$ are given (this case corresponds to phase transition in a particle at temperature $\boldsymbol{T}_{\boldsymbol{p}}$ ). Boundary conditions are the same as (2). The solution for both cases are obtained analytically. For the first case we have:

$$
\begin{array}{ccc}
T_{f}(r)-T_{\infty}=\frac{q}{6 \kappa_{f}}\left(a^{2}-r^{2}\right)+\frac{a^{2} q}{3 \kappa_{f}}\left(1+\frac{G \kappa_{f}}{a}\right), & (r<a) \\
T_{f}(r)-T_{\infty}=\frac{a^{3} q}{3 \kappa_{f}} \cdot \frac{1}{r}, & (r>a)
\end{array}
$$

For the second case the temperature distribution in fluid is given below:

$$
T_{f}(r)-T_{\infty}=\frac{\left(T_{p}-T_{\infty}\right)}{\left(G \kappa_{f} / a+1\right)} \frac{a}{r} .
$$

In both cases considered above, the effect of the KITR is characterized by the dimensionless parameter $\gamma=G \kappa_{f} / a$ expressed in terms of the interfacial resistance, radius of the particle, and thermal conductivity of the fluid. Figure 2 shows the dependences of the temperature of the particle at a given thermal flux and the thermal flux at a given temperature difference between the particle and 
infinity as a function of the parameter $\gamma$. The temperature and thermal flux are normalized to the values in the absence of the Kapitza temperature jump.

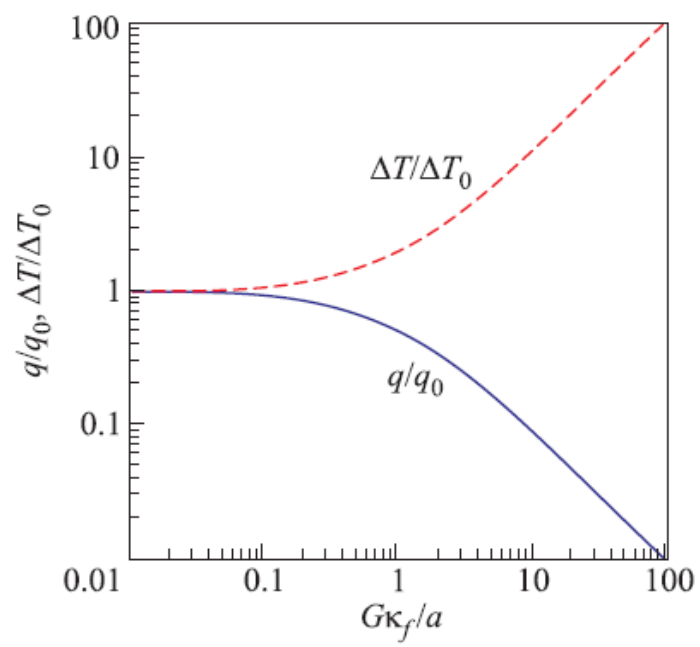

Figure2. Thermal flux (at given temperatures) and the temperature at the boundary of the particle at a given thermal flux versus the Kapitza temperature jump.

\section{Conclusions}

As calculations show, thermal processes in nanofluids can be characterized by the dimensionless parameter $\gamma=G \kappa_{f} / a$ expressed in terms of interfacial resistance, the radius of the particle, and thermal conductivity of the fluid. The general character of this parameter makes it possible to treat it as a similarity parameter when describing thermal processes in nanofluids with allowance for the Kapitza temperature jump.

The analysis of formulae (5) shows that the thermal conductivity of a nanofluid could be less than the thermal conductivity of a base fluid even when the thermal conductivity of particles is considerably higher than the thermal conductivity of the fluid (see Figure 1).

According to Figure 2, the Kapitza temperature jump should be taken into account when the parameter $\gamma>1$. It is worth noting that nanoparticles used both in hyperthermia and at the intensification of heat transfer owing to the heat of a phase transition are particles in shell (core-shell). In this case, there are two contact surfaces and two Kapitza temperature jumps correspondingly. Furthermore, the finiteness of the shell also contributes to additional thermal resistance. For this reason, the total contribution can be significant when solving particular problems.

Let us make an important remark with respect to thermal processes in nanofluids and KITP. To get stable nanofluid with respect to coagulation of lyophobic nanoparticles different surfactants are used. Molecules of the surfactant create some shell around particles. As it is shown in [15], the contribution of this shell with thickness $\delta$ and thermal conductivity $\kappa_{s}$ on thermal processes is the same as the value of the Kapitza temperature jump with parameter $G=\delta / \kappa_{s}$.

\section{Acknowledgement}

This work was supported by the Russian Science Foundation; Project no. 14-19-01379. 


\section{References}

1. P. L. Kapitza, Zh. Eksp. Teor. Fiz. 11, 1 (1941).

2. I. M. Khalatnikov, Theory of Superfluidity (Nauka, Moscow, 1971) [in Russian].

3. D. G. Cahill, W. K. Ford, K. E. Goodson, G. D. Mahan, A. Majumdar, H. J. Maris, R. Merlin, and S. R. Phillpot, J. Appl. Phys. 93, 793 (2003).

4. H.-K. Lyeo and D. G. Cahill, Phys. Rev. B 73, 144301 (2006).

5. C.-W. Nan, R. Birringer, D. R. Clarke, and H. Gleiter, J. Appl. Phys. 81, 6692 (1997).

6. Z. Ge, D. G. Cahill, and P. V. Braun, Phys. Rev. Lett. 96, 186101 (2006).

7. S. U. S. Choi, in Developments and Applications of Non-Newtonian Flows, Fluids Engineering Division FED, Vol. 231, Materials Division MD, Vol. 66 (ASME, New York, 1995), 99.

8. J. Buongiorno, D. C. Venerus, N. Prabhat, Th. McKrell, J. Townsend, R. Christianson, Y. V. Tolmachev, P. Keblinski, L.-W. Hu, J. L. Alvarado, I. Ch. Bang, S. W. Bishnoi, M. Bonetti, F. Botz, A. Cecere, et al., J. Appl. Phys. 106, 094312 (2009).

9. J. C. Maxwell, A Treatise on Electricity and Magnetism, 2nd ed. (Clarendon, Oxford, UK, 1881).

10. S. Laurent, S. Dutz, U. O. Hafeli, and M. Mahmoudi, Adv. Colloid Interface Sci. 166, 8 (2011).

11. I. Sharifi, H. Shokrollahi, and S. Amiri, J. Magn. Magn. Mater. 324, 903 (2012).

12. D. A. Scott, A. Lampureux, and B. R. Baliga, ASME J. Heat Transfer 135, 011013 (2013).

13. W. Wu, H. Bostanci, L. C. Chow, Y. Hong, S. J. Ding, M. Su, and J. P. Kizito, ASME J. Heat Transfer 135, 052202 (2013).

14. Landau L.D., Lifshitz E.M. Fluid Mechanics, (Pergamon Press, 1987).

15. Sukhinin G.I., Serebryakova M.A. and Novopashin S.A. J. Heat Transfer (2016). 